A N N A L E S Annales de Bretagne et des Pays de l'Ouest

\title{
L'engagement civique des notables urbains à Bourges (1480-1559)
}

\section{Alain Collas}

\section{OpenEdition}

\section{Journals}

Édition électronique

URL : http://journals.openedition.org/abpo/1112

DOI : $10.4000 / a b p o .1112$

ISBN : 978-2-7535-1499-7

ISSN : 2108-6443

Éditeur

Presses universitaires de Rennes

Édition imprimée

Date de publication : 20 septembre 2005

Pagination : 7-17

ISBN : 978-2-7535-0201-7

ISSN : 0399-0826

\section{Référence électronique}

Alain Collas, "L'engagement civique des notables urbains à Bourges (1480-1559) », Annales de

Bretagne et des Pays de l'Ouest [En ligne], 112-3 | 2005, mis en ligne le 20 septembre 2007, consulté le 01 mai 2019. URL : http://journals.openedition.org/abpo/1112 ; DOI : 10.4000/abpo.1112 


\title{
L'engagement civique des notables urbains à Bourges $(1480-1559)$
}

\author{
Alain COLLAS \\ Docteur et agrégé d'Histoire
}

Les notables urbains de la fin du Moyen Âge construisent leur fortune en même temps qu'ils contribuent à la prospérité de leurs villes. L'idée communément admise est que, au fur et à mesure de leur ascension sociale, ils se désintéresseraient progressivement des honneurs urbains, trop fades et peut-être trop éloignés du modèle nobiliaire ${ }^{1}$. Cela semble évident pour le sommet de la notabilité urbaine, les quelques familles qui dans chaque grande ville du royaume parviennent au fait des honneurs : le Parlement de Paris, le corps des notaires et secrétaires du roi, une prébende dans le prestigieux chapitre de Notre-Dame de Paris. Qu'advient-il des autres, de l'immense majorité de ceux que nous pourrions qualifier de notables ordinaires ${ }^{2}$ ?

La ville de Bourges apparaît exemplaire pour qui veut étudier ces notables. En effet, l'histoire de Bourges à la fin du Moyen Âge est celle d'une lente descente aux enfers. Le Duc Jean la dote dans les dernières années du XIV ${ }^{\mathrm{e}}$ siècle des éléments commerciaux et structurels qui doivent lui permettre de jouer un rôle de tout premier plan. La venue du Dauphin en 1418 peut même en faire une capitale. Dès 1425, le Dauphin quitte Bourges pour Tours emmenant avec lui les principales familles marchandes de la ville, notamment les Allégret. Jacques Cœur, Berruyer, construit ses affaires ailleurs, en Touraine ou en Languedoc, mais entraîne hors du Berry une autre partie des familles marchandes de Bourges ${ }^{3}$. L'adhésion à la Ligue du

1. Chevalier, Bernard, Les bonnes Villes de France du XIve au XVIe siècle, Paris 1982, notamment p. 147-148.

2. Les idées développées dans cet article font suite à une conférence donnée au Centre d'Études Supérieures de la Renaissance de Tours, le 5 mars 1996.

3. Voir pour l'essentiel CHEVALIER, Bernard, Tours ville royale, 1356-1520, Paris/Louvain 1975 p. 233 sqq. et MolLat, Michel, Jacques Coeur ou l'esprit d'entreprise, Paris 1988. Un bel exemple de marchand berruyer faisant fortune et carrière ailleurs, dans BREJON DE LAVERGNIE, M. E., "Guillaume de Varye et le commerce au Xve siècle ", dans Finances, pouvoirs et mémoire, hommages à Jean Favier, Paris 1999, p. 513-523. 
Bien Public dresse Louis XI contre Bourges. Il gardera tout au long de son règne une grande méfiance à l'égard de la capitale berrichonne. Enfin, un désastreux incendie détruit durablement la ville en juillet 1487 et met fin pour toujours à ses possibilités d'émerger en capitale économique.

Dans ce contexte, à la charnière $\mathrm{du} \mathrm{XV}^{\mathrm{e}}$ siècle et $\mathrm{du} \mathrm{XVI} \mathrm{I}^{\mathrm{e}}$ siècle, trois enjeux importants mobilisent les énergies des berruyers : le maintien des foires, la reconstruction de la ville après l'incendie de 1487 et la mise en place et le développement de l'Université. C'est par rapport à ces enjeux qu'il nous faut chercher à connaître le comportement des notables et tout particulièrement l'attitude des membres des grandes familles qui détiennent depuis parfois des décennies le pouvoir donné par les offices royaux et la fortune issue de la marchandise (les familles Le Roy, Lallemant, Bochetel, Chambellan, Fradet, Pelourde, du Breuil, de Perelles, Sardé, Pain, Tuillier...). Ces familles qui peuvent être réparties sur des cercles concentriques selon leur puissance et leur fortune, se renouvellent constamment au $\mathrm{xvi}^{\mathrm{e}}$ siècle par intégration de nouvelles familles ${ }^{4}$, notamment de marchands étrangers s'installant à Bourges comme les Vulcob venus de Flandres $^{5}$, ou les Salvi venus d'Italie ${ }^{6}$.

\section{Une participation constante à l'échevinage}

Le premier cercle de la notabilité berruyère du $\mathrm{XV}^{\mathrm{e}}$ siècle est composé des familles Le Roy, Lallemant, Bochetel. Parmi eux, seuls les Le Roy sont totalement absents des affaires de la ville. Nous ne les trouvons jamais mêlés aux grands événements ni en 1465, ni en 1474, ni plus tard durant les conflits religieux. Nous ne les trouvons jamais dans l'échevinage. Nous ne les trouvons d'ailleurs que très rarement dans les archives départementales. Ils répondent parfaitement au modèle des grands notables tournés vers la Cour, le Roi et le Royaume. Nous ne les rencontrons à Bourges que lorsqu'ils y viennent pour régler leurs affaires privées et s'occuper de leur patrimoine, soubassement de leur fortune et de leur place sociale. Patrimoine qui se situe d'ailleurs hors des murs de la ville ${ }^{7}$. En Berry, ils vivent en gentilhommes. Ainsi, Jacques III Le Roy, receveur général des Finances Outre Seine, qui fait faire des travaux dans sa maison située au cour d'une métairie afin d'y habiter en $1496^{\circ}$.

4. Collas, Alain, "Les gens gui comptent à Bourges au Xve siècle ", Annales de Bretagne et des Pays de l'Ouest, 1994, tome 101, n 3, p. 49-68.

5. Arch. dép. du Cher, E $1721, \mathrm{f}^{\circ} 2800$.

6. RaYnAL, Louis, Histoire du Berry depuis les temps les plus anciens jusqu'en 1789, Bourges, 1844, tome 3.

7. À Saint-Florent-sur-Cher, à 15 kilomètres au sud-ouest de Bourges.

8. Le 14 avril 1496 après Pâques. Arch. dép. du Cher, E 1716, f ${ }^{\circ} 15$. Le Roy y est qualifié de " noble homme ". Il deviendra la même année notaire et secrétaire du roi (LAPEYRE, André et SCHEUREUR, Roger, Les notaires et secrétaires du roi (1461-1515), Paris 1978, p. 209, notice 425). 
En revanche, Les Lallemant, malgré leurs hautes fonctions, ne négligent pas les affaires urbaines. Jean II Lallemant l'aîné, receveur général de Normandie, est maire de Bourges en $1500^{9}$. Jean III Lallemant le jeune, son frère, Trésorier et receveur général du Languedoc, est maire en $1510^{10}$. La famille Bochetel, qui fournit de nombreux notaires et secrétaires puis des ambassadeurs en Italie, des humanistes, est très présente à Bourges. François I Bochetel, notaire et secrétaire du roi ${ }^{11}$ est échevin de Bourges en 1488 et 1490 . Il est maire en 1494-1495 et en 1498-1499 ${ }^{12}$. Bernardin II, son fils, est échevin en 1501-1502, maire de 1505 à 1507 et en 1530-1531 ${ }^{13}$. Les membres des autres cercles de la notabilité fournissent l'essentiel des maires de Bourges (les Chambellan en 1492, 1519, 1534, 1569; les Fradet en 1508 et 1536; les Pelourde en 1526; les du Breuil en 1500; de Perelles en 1508; les Pain en 1502 et 1546; les Tuillier en 1482, 1485 et 1488; les Houet en $1515 . ..)^{14}$.

La hiérarchie entre les notables est respectée puisque hormis Étienne Houet, les membres de ce que nous pourrions qualifier de quatrième cercle, sont massivement présents à l'échevinage mais sans jamais parvenir à la fonction de maire. Nos gens sont donc associés aux affaires de la ville par une participation constante à l'échevinage. Ils monopolisent la fonction de maire. Cela ne nous donne qu'une partie de la réponse à la question de leur investissement dans les affaires locales. Peut-être ne sont-ils là que pour les honneurs? Pour en savoir plus, il est nécessaire d'essayer de les regarder agir face aux problèmes essentiels que la ville doit affronter.

\section{La mesure de leur investissement local}

\section{La question des foires}

Le premier enjeu important pour Bourges à la fin du $\mathrm{Xv}^{\mathrm{e}}$ siècle est le maintien des foires. Les États de Tours de 1484 avaient décidé que les foires de Pâques et de la Toussaint seraient transférées de Lyon vers Bourges pour cinq ans. Au printemps 1485, le Conseil du roi décide d'envoyer deux commissaires enquêter sur les villes de Dijon, Châlons, Bourges et Lyon afin de savoir comment se déroulent les foires et d'examiner la possibilité de les maintenir toutes ou d'en supprimer certaines ${ }^{15}$.

Les deux commissaires commencent leur voyage en juin 1485, sont reçus par les édiles dijonnais, puis par ceux de Châlons, sans aucun problème. En septembre 1485, ils arrivent à Bourges. Ils ont alors la surprise

9. Ribault, Jean-Yves, " Notes sur les origines de la famille Lallemant ", Cahiers d'archéologie et d'histoire du Berry, $\mathrm{n}^{\circ} 29$, juin 1972, p. 62-64.

10. Ibidem.

11. LAPEYRE, André, Scheureur, Roger, op. cit., notice 69.

12. Jongleux, Henri, Les Archives de la ville de Bourges avant 1790, publication des archives municipales, p. 28-33.

13. LAPEYRE, André, SCHEUREUR, Roger, op. cit., notice 68.

14. JongleuX, Henri, Les Archives de la ville de Bourges..., op. cit.

15. Pour la suite voir le texte transcrit dans RAYNAL, Louis, op. cit., p. 538 sqq. Nous adoptons cette transcription. 
d'être accueillis par une foule d'habitants, artisans, apprentis, armés de bâtons, de couteaux, qui les menacent et les enferment dans leur chambre. Ils sont encore plus surpris de voir à la tête de cette émeute populaire, Guillaume Sardé, lieutenant général du bailli, Pierre Dubreuil, avocat du roi, Guillaume Pain, procureur du roi, Claude Pelourde, conservateur des privilèges de la foire et Pierre Tuillier, maire de la ville. Le ton monte, les épées sortent et Claude Pelourde exige que les commissaires lui remettent l'ensemble des papiers qu'ils avaient rédigés sur les foires de Dijon et de Châlons. Les commissaires refusant d'obtempérer, les officiers du roi forcent les coffres et prennent de force les manuscrits. Les commissaires s'adressent alors au lieutenant général,

" lui disant que estions fort esbahys comme luy, qui devait craindre son honneur et sa conscience, souffroit telles choses estes faites [...] et comme il voit faire un tel abus et si dangereux par commocion et assemblée de peuple. [Ils continuent ainsi :] Nous voulumes gracieusement remonstrer auxdits officiers le péril et dangier que povoit estre en ceste matière et que ils voyaient la grant rébellion et désobéissance qui se faisait grant irreverence et mespris de l'auctorite du Roy. Les sommant et requerant et faisant commandement de par ledit seigneur de nous aider, assister et procurer a nous deffendre de l'oppression du peuple qui par là estoit et quils fissent retirer la multitude des gens de la ville».

Le lieutenant général répond alors « quil ny povoit remedier contre eulx et que les gens de la ville le requeroient et pressoient et quil falloit quit le fist " et les officiers du roi menacent de mettre les commissaires du roi en prison.

Cet épisode nous permet de voir les officiers royaux du bailliage prendre parti pour la ville de Bourges et les habitants de la ville contre les envoyés du roi. Ils justifient cette action par la nécessité de défendre les intérêts des berruyers et l'obligation dans laquelle ils sont de le faire. Dans une circonstance où la tension montre la vivacité de l'inquiétude des habitants de Bourges quant à l'avenir économique de leur ville, les officiers du roi trahissent leur mission pour le moins, considérant que les impératifs de la ville et des habitants sont plus forts que ceux du pouvoir qu'ils sont censés incarner. Nos hommes en l'occurrence semblent faire preuve de patriotisme municipal. Il est particulièrement intéressant de constater que pour deux d'entre eux, ils n'en sont pas à leur première manifestation. En effet, Guillaume Sardé a été arrêté par ordre de Louis XI le 27 avril 1474 comme responsable de l'émeute qui avait soulevé le peuple de Bourges contre une taxe. Pierre Tuillier, alors avocat fiscal, avait été inquiété pour la modération de son attitude face aux émeutiers lors de cette affaire et avait été révoqué ${ }^{16}$.

Le groupe qui défend les intérêts de Bourges face aux commissaires royaux en 1485 est hétéroclite. Guillaume Sardé, Guillaume Pain, Pierre

16. RivaUD, David, "La destatable commocion de Bourges (23 avril 1474) et son règlement. Utilisation politique de l'autorité royale dans un conflit urbain ", Cahiers d'archéologie et d'histoire du Berry, n 141, mars 2000, p. 3-12. 
Tuillier appartiennent au troisième cercle de la notabilité. Guillaume Sardé devient lieutenant particulier en 1465 avant d'être lieutenant général ${ }^{17}$. C'est le premier officier important d'une famille qui jusqu'alors est peu parue dans la ville. Un Simon Sardé émerge tristement des archives en obtenant en 1453 une lettre de rémission pour le meurtre d'un étudiant ${ }^{18}$. Guillaume est accompagné en 1485 par son fils Jean qui deviendra son lieutenant particulier. Guillaume est condamné le 20 mars 1486 par le Conseil du roi (avec ses compères Guillaume Pain, Pierre Dubreuil et Claude Pelourde) à payer une amende de 1000 marcs d'argent. II continuera sa carrière d'officier du roi dans les procès, de destitution par le bailli en réintégration par le Parlement de Paris jusqu'en $1493^{19}$. La famille Sardé ne sort pas de la notabilité marchande de Bourges jusqu'à la fin du XVI ${ }^{\mathrm{e}}$ siècle, s'alliant en 1506 avec les Salvi, marchands italiens fraîchement arrivés à Bourges. Pour cette famille, le maintien des foires et les retombées économiques qu'elles génèrent sont de toute première importance.

Les Tuillier sont aussi avant tout des notables marchands tout au long du XV ${ }^{\mathrm{e}}$ siècle ${ }^{20}$. Quant aux Pain, famille presque inconnue au Xve siècle, ils apparaissent avec Guillaume, procureur du roi en 1485 qui transmettra cet office à son fils Gilles en $1502^{21}$. Ils fournissent de nombreux échevins au $\mathrm{XVI}^{\mathrm{e}}$ siècle (Denis, Étienne, Jean), mais sans plus. Pour eux le service de la ville, la défense des habitants, c'est une façon d'avoir du pouvoir, une part de puissance.

Claude Pelourde et Pierre Dubreuil appartiennent à des milieux un peu différents, à un cercle plus relevé. La présence de Claude Pelourde à la tête de cette " émotion " de 1485 est même tout à fait intéressante à souligner au-delà de son office. Les Pelourde sont en effet une vieille famille de Bourges. Le premier membre que nous rencontrons dans un office est Laurent Pelourde, garde du scel de la prévôté en $1283^{22}$.

Fils de Guillaume III Pelourde, échanson du roi, Claude a un frère, Jean VII, secrétaire du duc de Guyenne, un autre, Guillaume VI, chanoine à Saint-Pierre Le Puellier de Bourges et un beau-frère qui n'est autre que François I Bochetel ${ }^{23}$, notaire et secrétaire du roi ${ }^{24}$. Nous pouvons même supposer qu'il est bien placé pour avoir des raisons de craindre le vrai objet

17. DUPONT-FERRIER, Gustave, Gallia Regia -État des officiers des bailliages de 1328 à 1515, tome I, Paris, 1942, p. 3901.

18. Arch. nat., JJ 185 acte 301 f 2080 . Un Nicolas Sardé est garde du scel de la prévôté de Bourges en 1392-1393. René Lacour, Le gouvernement de l'apanage de Jean, duc de Berry, annexe XIX et Arch. nat., JJ 188 A, n 51 ; JJ 185 A, n 32.

19. De très nombreux actes, par exemple Arch. nat., $X^{1 a} 1496$, p. 329.

20. Sur les autres membres de la famille Tuillier voir notamment : Michel MolLat (dir.), Les affaires de Jacques Coeur, Journal du Procureur Dauvet, 2 vol., Paris 1952-1953, et Arch. dép. du Cher, E $222, \mathrm{f}^{\circ} 88 \mathrm{v}^{\circ}$.

21. Arch. dép. du Cher, E $1716, \mathrm{f}^{\circ} 281 \mathrm{v}^{\circ}$.

22. DevalLLY, Guy, Le Berry du X $X^{e}$ au XIII ${ }^{e}$ siècle, Paris-La Haye 1973, p. 532-533.

23. On peut suivre la généalogie de Girard de Villesaison (Arch. dép. du Cher, 2 F 259 $\mathrm{n}^{\circ} 167$ ) puisqu'elle est confirmée (Arch. dép. du Cher, E 1717, $\mathrm{f}^{\circ} 620$ ).

24. LAPEYRE, André, SCHEUREUR, Roger, op. cit., notice 69. 
de la mission des envoyés royaux, concernant l'avenir des foires. Pierre Dubreuil I, petit fils, fils et frère de notaires et secrétaires du roi, appartient au même monde ${ }^{25}$, même s'il s'agit du cadet condamné à voir s'éloigner de lui la branche aînée aspirée par les honneurs. Nous avons bien là quelques exemples de grandes familles qui semblent s'intéresser de très près, sans hésiter à s'engager, aux affaires de la ville, aux côtés d'autres notables moins huppés et au milieu (à la tête?) de la population.

Si nous suivons Bernard Jarry ${ }^{26}$, la défense des foires fut l'occasion à bien d'autres familles berruyères de s'engager de manière plus feutrée, plus policée. En effet, il voit dans la Confrérie de la Table ronde, au-delà du lieu de convivialité fraternelle, une union ayant pour finalité de soutenir les intérêts de la ville de Bourges face à Lyon. Nous retrouvons parmi les " chevaliers » outre Guillaume Sardé, Jean Lallemant l'aîné, Jean Lallemant le jeune, Martin Chambellan, Charles Fradet, Michel de Cambray, mais aussi Jean Georges, l'un des fondateurs en mai 1486. Jean III Geôrges et Jean Lallemant l'aîné, qui furent par ailleurs échevins et maires de Bourges, créent la Confrérie de la Table ronde en la dotant de statuts dans lesquels il est mentionné comme premier but "l'exaltation de la ville de Bourges, croissance et augmentation de l'onneur et bruit des enfans manans et habitans en icelle ". Ces membres des tous premiers cercles de la notabilité qui fréquentent les honneurs parisiens et l'entourage royal n'ont manifestement pas tourné le dos à la ville qui a vu se développer l'assise de leur fortune.

Ces engagements en faveur des foires de Bourges, malgré le réseau d'influence de ces hommes, ne permet pas à la ville frappée par le terrible incendie de 1487, de garder cette activité essentielle au rayonnement commercial. Elle ne peut tenir les foires de la fin de l'année 1487. Un séjour de Charles VIII à Bourges, logé chez Guillaume Chambellan (dont la première épouse avait été Guillemette Lallemant), ne permet pas d'emporter la décision. Charles VIII décide le transfert définitif des foires à Lyon ${ }^{27}$.

\section{La reconstruction de la ville après l'incendie de 1487}

Le second combat, la reconstruction de la ville après l'incendie de 1487, voit aussi ces notables se mobiliser et s'engager. Dès 1488, nous trouvons nos familles à l'œuvre pour la reconstruction, à commencer par François I Bochetel, échevin, que les comptes municipaux montrent très actif, notamment pour l'acquisition d'un terrain permettant la construction d'un Hôtel de ville ${ }^{28}$. Non seulement dans ce cas il s'agit de reconstruire, mais encore d'asseoir le prestige de la fonction échevinale. Les Chambellan et les

25. Gallia Regia ..., op. cit., I, p. 3908 et LAPEYRE, André, ScheUREUR, Roger, op. cit., f ${ }^{\circ}$ XXXIX. 26. JARRY, Bernard, " Les statuts de la Table ronde de Bourges ", Cahiers d'archéologie et d'histoire du Berry, $\mathrm{n}^{\circ} 29$ juin 1972, p. 18-33.

27. Nos hommes continuent néanmoins de se battre pour ces foires puisqu'en février 1515, François $1^{\text {er }}$ est encore obligé de réaffirmer les droits de Lyon contestés par Bourges. Ordonnances des rois de France, François $1^{\text {er }}$, t. 1, pièce 25, p. 91.

28. Arch. mun. de Bourges, CC 262, CC 263. 
Bochetel fournissent tout au long des années 1490 les maires de la renaissance de la ville. Ils le font en même temps que la lutte pour le maintien des foires. Il s'agit dans l'un et l'autre cas de permettre à leur ville de retrouver au plus vite les conditions de la prospérité.

Nous y voyons une véritable manifestation de l'attachement à la ville car contrairement aux Sardé ou aux Tuillier, ces familles n'ont plus réellement le même besoin de la prospérité locale. Notaires et secrétaires du roi pour les Bochetel ou les Georges, Receveurs généraux de finances pour les Lallemant, alliés à des membres du Parlement de Paris pour les Chambellan, ils ont métairies et hôtels particuliers à la campagne (notamment à Neuvy sur Baranjon pour les Chambellan et les Georges). Ils font aussi des mariages qui les conduisent hors du Berry (le Picard, de Marie pour les Lallemant par exemple ${ }^{29}$ ), Ils sont pourvus de bénéfices à Notre-Dame de Paris ${ }^{30}$. Tout pourrait les aspirer hors de Bourges, hors du Berry. Tout montre au contraire qu'ils gardent un ancrage dans la ville où ils habitent.

\section{Le développement de l'université de Bourges}

Le dernier exemple que nous en donnerons est le rôle joué par ces familles notables pour le développement de l'Université de Bourges. À l'origine de cette création, on trouve déjà un membre d'une grande famille de Bourges. En effet, c'est Pierre Il Fradet, doyen du chapitre de Saint-Étienne de Bourges, qui s'active à Rome à partir de 1464 pour obtenir l'accord du Pape. Il meurt d'ailleurs à Rome en mission avant de voir l'installation à Bourges de l'Université le 9 mars $1467^{31}$. Pierre II Fradet, régent de l'Université d'Orléans ${ }^{32}$ prend ainsi position pour sa ville malgré l'opposition active des universités d'Orléans et de Paris.

Dans le contexte général des difficultés connues par Bourges à la fin du $\mathrm{XV}^{\mathrm{e}}$ siècle, l'Université décline rapidement. Les notables berruyers vont se mobiliser à partir des années vingt pour la redynamiser. Et nous retrouvons un Pelourde à la tête de ce nouveau défi. C'est Jean VIII, fils de Claude, l'émeutier de 1485, qui maire de Bourges en 1526 entreprend avec les échevins Jean de Cucharmois, Gabriel Labbé et Pierre Foucaut, un véritable tour

29. Le Picart, famille du Val de Marne et de l'Oise, de Marie, famille parisienne. LAPEYRE, André, SCHEUREUR, Roger, op. cit., notice 454, 408, 409.

30. Gabriel Pelourde, Guillaume Bochetel, Renaud Bochetel, David Chambellan notamment. Voir DERonne, Éliane, « Les origines des chanoines de Notre-Dame de Paris de 1450 à 1550 ", Revue d'Histoire Moderne et Contemporaine, tome XVIII, janvier-mars 1971, p. 1-29.

31. Lui-même docteur in utroque jure, Pierre II Fradet est le fils de Pierre I, capitaine de la grosse Tour de Bourges. Petit fils de Marguerite Chambellan, son frère aîné Jacquelin était capitaine de la grosse Tour de Bourges également. Pierre II fut reçu Conseiller clerc au Parlement de Paris le $1^{\text {er }}$ janvier 1460. Voir Édouard MAugis, Histoire du Parlement de Paris de l'Avènement des rois Valois à la mort d'Henri IV, 3 vol., Paris 1913-1914, t. 3, p. 96 et 162; et Dictionnaire d'histoire et de géographie ecclésiastique, vol. X, Paris, 1967, p. 181.

32. Le 22 juin 1460 : PrÉGuEZuElo, Joelle, Les chanoines de Saint-Étienne de Bourges à la fin du Moyen Âqe. Histoire d'un groupe social. Mémoire de maîtrise dactylographié (sous la direction de B. Chevalier) Université de Tours 1974, pièces justificatives III. 
du royaume pour trouver les professeurs de renom qui puissent relancer la réputation de l'université de Bourges ${ }^{33}$. Ils parviennent à faire venir Alciat à Bourges, enseigner le droit, ce qui va contribuer à transformer cette ville pour quelques années en centre culturel humaniste.

Non seulement nos gens ne se détournent pas des honneurs urbains, mais loin de se contenter seulement des titres et avantages apportés par l'échevinage, ils agissent et contribuent largement à maintenir Bourges à un certain rang jusqu'aux conflits religieux.

\section{Un comportement original des notables berruyers}

II y aurait donc, chez les notables de Bourges, un comportement différent de celui des tourangeaux étudiés par B. Chevalier. Essayons de comprendre pourquoi. Nous avons évoqué une seule grande famille de Bourges tournant le dos à la ville : Le Roy. Alliée aux Briçonnet, cette famille est implantée dans la proximité de la Cour, dès le règne de Charles VII ${ }^{34}$. Jacques I Le Roy était écuyer tranchant du duc Jean de Berry. Or, nous trouvons d'autres personnages quittant Bourges dans les mêmes années. Adam Rolant, fils d'un médecin du duc de Berry, devient notaire et secrétaire du roi dès 1441. S'il reste propriétaire d'une maison à Bourges, il possède, dans les années 1460, maisons et terres à Tours. Son fils Jacques, lui-même notaire et secrétaire du roi, n'apparaît plus à Bourges mais nous le trouvons seigneur de la commune de Monts près de Tours ${ }^{35}$. Nous pourrions encore citer Jacques II Bourdin, né à Bourges, notaire et secrétaire du roi également, qui achète ses seigneuries dans la région parisienne et dont les enfants sont parisiens ${ }^{36}$.

Toutes ces familles ont en commun d'avoir été, dès le règne de Charles VII, aspirées par les honneurs. Elles ont suivi le roi en Touraine ainsi que la trajectoire des familles tourangelles qu'elles fréquentent, Briçonnet, Ruzé ou autre de Beaune. Elles se sont enrichies à la cour et ont très vite voulu s'intégrer à un mode de vie nobiliaire en même temps qu'elles parvenaient aux plus hautes fonctions. Le même mouvement a été étudié au XIV ${ }^{\mathrm{e}}$ siècle lorsque la cour est à Paris ${ }^{37}$. Pour les notables de

33. RaYnAL, Louis, Histoire du Berry..., op. cit., t. III, p. 370 et 399. Jean VIII Pelourde est depuis 1504 au moins lieutenant à la Conservation des privilèges royaux de l'Université (Arch. dép. du Cher, E 1717, f ${ }^{\circ}$ 620). Gabriel, chanoine à Notre-Dame de Paris est son frère. Jean Cucharmois appartient à une riche famille de marchands récemment arrivés de Lyon.

34. Arch. dép. du Cher, 2 F 255 n 15 et LAPEYre, André et Scheureur, Roger, op. cit., notice 425 .

35. LAPEyre, André, Scheureur, Roger, op. cit., notice 598 et 600 et Mollat, Michel (dir.), Les affaires de Jacques Cour, Journal du Procureur Dauvet, op. cit., I, p. 148.

36. LAPEYRE, André, ScheUREUR, Roger, op. cit., notice 86.

37. Voir notamment BovE, Boris, "Espace piété et parenté à Paris aux XIII et XIV ${ }^{\mathrm{e}}$ siècles d'après les fondations d'anniversaires des familles échevinales ", dans Religion et société urbaine au Moyen Âge. Études offertes à Jean Louis Biget, Publications de la Sorbonne, 2000, p. 253-281. 
Bourges, le facteur qui favorise cette ascension précoce c'est la présence à la cour du duc Jean, puis la présence du Dauphin.

Les grandes familles que nous étudions, qui sont à Bourges après 1461, prennent leur essor dans un autre contexte. Les Pelourde et les Le Roy apparaissent dans les archives dans les mêmes années, à la fin du XIII ${ }^{\mathrm{e}}$ siècle, mais les premiers sont encore lieutenants particuliers du roi lorsque les seconds sont auprès de Charles VII. Jean Il Lallemant est encore qualifié de "prudent homme " en 1467 quand Jean Briçonnet l'aîné est déjà receveur du Languedoil. Les Bochetel commencent à cumuler les fonctions importantes avec le règne de Louis XI. Ils font figure de précurseurs dans le groupe que nous étudions. Ce qui trouble l'image, c'est que ces familles sont alliées. Les Chambellan, les Bochetel, les Lallemant, les Georges, les Pelourde et les Le Roy ont à un moment ou à un autre marié leurs enfants. Les Le Roy s'allient quatre fois avec les Pelourde, trois fois avec les Lallemant, deux fois avec les Chambellan, une fois avec les Bochetel et une fois avec les Briçonnet. Mais ces mariages n'ont pas la même signification. Marie Briçonnet, fille de Jean Patron, épouse Jacques Le Roy, receveur comme son frère François. C'est une alliance entre égaux du même monde. Lorsque Nicole Chambellan épouse Jean Le Roy, elle permet à sa famille de monter une marche ${ }^{38}$. Cette alliance se termine d'ailleurs en contestation et en procès autour de la restitution de la $\operatorname{dot}^{39}$.

Nous avons le sentiment qu'il y a plusieurs générations de notables à Bourges. La première génération, celle des Le Roy, des Rolant... qui surent très tôt profiter de l'importance du duc Jean pour bâtir une stratégie d'ascension rapide qui va les conduire dans l'entourage de Charles VII, en Touraine, va quitter assez vite la marchandise et le Berry. La seconde génération va construire une ascension beaucoup plus lente. Certains sont présents dès le XIV ${ }^{\mathrm{e}}$ siècle, mais n'ont pas su ou pas pu profiter de la cour du duc Jean. Ainsi, les Pelourde ont-ils subi les contre coups d'une affaire de coups et blessures d'un avocat du roi, Renaud Fournier, qui se termine par un meurtre dans la deuxième moitié du Xv $\mathrm{siècle}^{40}$. Alors que ceux de la première génération ont déjà tourné le dos à Bourges, ceux de la deuxième génération accèdent à leur tour à de hautes fonctions. Mais lorsque Louis XI parvient au pouvoir, ils sont encore à Bourges ce qui sous ce règne n'est pas une condition favorable et ils ne vont vraiment émerger qu'avec le règne de Charles VIII.

Or, si les fonctions exercées par les uns et les autres sont semblables, les comportements sont différents. Guillaume II Bochetel, secrétaire des finances, fait partie du Conseil étroit dès l'arrivée sur le trône d'Henri II ${ }^{41}$. Sa famille est au sommet comme l'avait été auparavant les Briçonnet ou de

38. CHEvalier, Bernard, Tours, ville royale, 1356-1520, Paris/Louvain, 1977 pour les Briçonnet et Arch. nat., X1A 1496, f $^{\circ} 279$ pour l'alliance Le Roy-Chambellan.

39. Arch. nat., X1A $1496, \mathrm{f}^{\circ} 319 \mathrm{v}^{\circ}$.

40. Arch. nat., JJ 82, f 277 et $\mathrm{f}^{\circ} 290$, Arch. nat., JJ 81, $\mathrm{n}^{\circ} 725, \mathrm{f}^{\circ} 37 \mathrm{v}^{\circ}$.

41. Cloulas, Ivan, Henri II, Paris, 1983, p. 146 et p. 520. 
Beaune. Mais elle reste attachée à la ville de ses origines. Guillaume III et Jean III Bochetel sont au chapitre cathédrale de Bourges, investis dans l'Université locale. Bernardin II, nous l'avons vu, est maire. Tous ont en commun un regard tourné vers le modèle nobiliaire, une intense activité d'achats de seigneuries, de terres, de métairies. Tous ont le souci de vivre à la manière des nobles, d'en épouser les membres cadets. Mais autant les premiers, ceux qui réussissent à émerger avant les années 1450, coupent les liens avec leurs racines et se fondent peu à peu comme ce Jacques Rolant, écuyer, seigneur de la Cigogne, autant les seconds restent attachés à leurs origines géographiques, à la ville qui les a vus naître. Ce sont ceuxlà qui constituent le "quatrième estat " décrit par Montaigne vers $1580^{42}$. Une fois la cour royale remontée vers le nord, les familles tourangelles de la deuxième génération se comporteront comme celles que nous étudions à Bourges. Les Briçonnet, Berthelot, Lopin étant partis, la Touraine verra s'enraciner dans les affaires de la ville et dans les échevinages, les Binet, Chalopin ou Cottereau. Et cet attachement, cet investissement sera durable, bien au-delà de la Renaissance ${ }^{43}$.

À Bourges, toutes les grandes familles qui restent dans la ville après 1461 sont actives dans la gestion des affaires et dans les secousses des guerres de religion jusqu'à la fin du Xvi ${ }^{\mathrm{e}}$ siècle. Les Chambellan, les Pelourde, les Fradet, les Bouer, les Bochetel, agiront ensemble ou les uns contre les autres à la tête de la ville durant la Ligue. Ils le feront alors aux côtés d'une troisième génération de notables urbains qui émergent au début du $\mathrm{XVI}^{\mathrm{e}}$ siècle, les Girard, Les Vulcob, les Mareschal. La notabilité urbaine de Bourges est en continuelle regénérescence. Mais, à partir du règne de Louis XI qui apparaît bien comme une période charnière, ses membres restent où que les conduisent leurs fonctions, attachés à Bourges et au Berry. L'importance des crises (locales comme l'incendie de 1487 ou générales comme les guerres de religion), ne permettent plus les ascensions fulgurantes ni l'abandon ou le détachement des terres d'origine et du soubassement de la fortune.

42. COLLAS, Alain, " Entre noblesse et bourgeoisie un modèle social au Xve siècle les officiers du roi ", dans Le modèle à la Renaissance, sous la direction de Jean LAFOND, Paris, Vrin, 1986, p. 91-100.

43. M. Gilles Bertrand Cottereau est maire en 1718 à Tours, 193 ans après son ancêtre Guillaume. Jérome Binet occupe le fauteuil de maire en 1600, après son ancêtre Jean qui l'avait occupé en 1524. Les Cottereau donnent six maires à Tours en près de deux siècles. Voir CheValier, Bernard, Histoire de Tours, Toulouse, Privat 1985, p. 403-406. 


\section{RÉSUMÉ}

Les notables de Bourges ne semblent pas avoir le comportement souvent décrit de distanciation par rapport à la ville, de leurs origines au $\mathrm{XVI}^{\mathrm{e}}$ siècle. Bien au contraire, face aux grands enjeux de Bourges (reconstruction après l'incendie de 1487, maintien des foires, développement de l'université), ils apparaissent particulièrement engagés malgré les hautes fonctions qui les conduisent à la Cour.

Cela ne serait-il pas le fait d'une deuxième génération de notables accédant aux honneurs à partir du régime de Charles VIII et aux stratégies différentes de leurs aînés?

\section{ABSTRACT}

It seems that the often described behaviour of distancing as regards their city, and this from their origins up to the 16th century, don't fully apply to the notables of Bourges.

Quite the opposite, for, in spite of the high charges that would lead them to the Court, the notables seem particularly involved in the great challenges of Bourges; i.e. the reconstruction after the fire of 1487, the upholding of the fairs and the expansion of the university.

Couldn't that be the fact of a second generation of notables who, attaining to the honours since the beginning of the reign of Charles VIII, would adopt a different strategy from that of other elders? 